\title{
ESTUDO E MEDIDA DA DISPERSÃO DE MODOS DE POLARIZAÇÃO E SUA DEPENDÊNCIA COM A TEMPERATURA
}

\author{
Rafael Auad Cardoso \\ Andrés Pablo López Barbero ${ }^{2}$ \\ Janaina Ferreira ${ }^{3}$ \\ Alexandre Bessa ${ }^{4}$ \\ Ricardo Marques Ribeiro ${ }^{2}$
}

Resumo: O presente trabalho investiga o impacto da dispersão por modos de polarização (PMD) em uma fibra óptica compensadora de dispersão (DCF). Vale ressaltar que este fenomeno é um fator limitante nos sistemas de alta capacidade ainda pouco explorado e, por ser de natureza aleatória, difículta sua determinação. Desta forma, torna-se necessário recorrer a modelos estastísticos. Foram utilizados dois métodos de medidas da PMD: O primeiro baseado na técnica interferométrica no domínio temporal e o segundo na técnica polarimétrica no domínio da frequência.

Palavras-Chave: Dispersão por modos de polarização (PMD), Fibra compensadora de dispersão, Técnica polarimétrica e Técnica interferométrica

\begin{abstract}
This study investigates the impact of polarization mode dispersion (PMD) in a dispersion compensation optical fiber. It is important to highlight that the phenomenum is a major limiting factor and the random fact difficulty its determination. In this way, statistical models are necessary for this purpose. Two different methods of PMD determination were used: The first one is based on interferometry in the time domain and the second, the polarimetric technique in the frequency domain.
\end{abstract}

Keywords: Polarization mode dispersion (PMD), Dispersion compensation fiber optics, polarimetric technique, interferometric technique.

\section{INTRODUÇÃO}

Com a evolução dos sistemas ópticos de longa distância e a crescente necessidade de aumento das taxas de transmissão de dados, o mercado de telecomunicaçôes evidênciou à necessidade de meios de transmissão mais adequados. Até algum tempo atrás (Cerca de 12 anos), o enfoque das grandes operadoras no Brasil e no mundo estava voltado para redes de média e longa distância, porém, desta maneira tinha-se uma grande capacidade de transmissão de longa distância ociosa.
Então, o rumo mudou e passou a se prestar mais atenção no mercado metropolitano, o qual alimentou o mercado interurbano e rapidamente utilizou toda capacidade de banda disponível nos trechos de longa distância.

Os principais fatores limitantes da taxa de transmissão nos enlaces ópticos são: A atenuação, a dispersão do sinal e os efeitos não-lineares. Para contornar os efeitos não-lineares, busca-se operar com potências ópticas em níveis onde os efeitos são aceitáveis. Para o efeito da atenuação, onde os métodos de amplificação estão comercialmente bem

\footnotetext{
1 Dir de atendimento a Claro, Embratel, rfac@embratel.com.br

2 Dep. de Engenharia de Telecomunicaçōes, Universidade Federal Fluminense, pablo@uff.com.br

3 Departamento de comunicações ópticas, Inmetro, janaina78@gmail.com

${ }^{4}$ Dep. de Engenharia de Telecomunicações, Universidade Católica de Petrópolis, alexandre.bsantos@ucp.br
} 
definidos e plenamente utilizados (GALTAROSSA, 2005), o problema torna-se satisfatoriamente controlado. A dispersão do sinal numa fibra monomodo é a combinação simultânea da dispersão cromática e da dispersão de polarização, PMD. A dispersão cromática, que é fortemente dependente com comprimento de onda e da largura espectral da fonte, pode ser dividida em dispersão material (dependência do índice de refração do material com o comprimento de onda) e em dispersão de guia de onda (dependência da constante de propagação com o comprimento de onda) em função do perfil de índice de refração da fibra. A dispersão dos modos de polarização (PMD) é causada pela variação aleatória da birrefringência da fibra monomodo, que altera a constante de propagação dos dois estados de polarização. A aleatoriedade da PMD depende também do comprimento de onda. A origem da PMD tem diversos fatores, tais como: assimetria geométrica e estresse (pressões e tensões mecânicas) existentes nas fibras. O primeiro fator pode ser reduzido durante o processo de fabricação. Mas as tensões externas, que dependem das condições climáticas e tensões mecânicas, fazem da PMD um processo aleatório e intrínseco dos sistemas de transmissão.

Desta forma, torna-se necessário um método eficiente para se compensar e controlar tal fenômeno aleatório. O elemento essencial para o controle da PMD é sua própria medição, que serve para permitir o controle de qualidade e a introdução de melhorias nos processos de fabricação das fibras e cabos. A medida da PMD também permite avaliar enlaces instalados e verificar sua compatibilidade com os parâmetros requeridos nos sistemas ópticos de alta capacidade. Atualmente existem diversos métodos para a medida da PMD. No entanto, a natureza aleatória desta grandeza torna difícil sua caracterização. Consequentemente, um diagnóstico (medida) e a compensação da PMD não consiste de uma simples operação. Pelo contrário, faz-se necessário uma análise estatística detalhada dos dados coletados em campo e, para sua compensação, a utilização de diversos componentes ópticos.

\section{TEORIA DA PMD}

Nas fibras ópticas padrão G652, o modo fundamental é o $\mathrm{HE}_{11}$. Esse modo de propagação admite dois estados de polarização ortogonais, conhecidos como PSPs (Principal states of polarization), os quais, numa fibra óptica ideal, são degenerados. A presença de birrefringência na fibra causa a quebra da degenerescência entre os modos de polarização e, conseqüentemente, uma diferença de velocidade de grupo entre estes, gerando um atraso de grupo diferencial (DGD), simbolizado por $\Delta \tau$. Na Figura 1 é possível visualizar a representação do atraso de grupo diferencial.

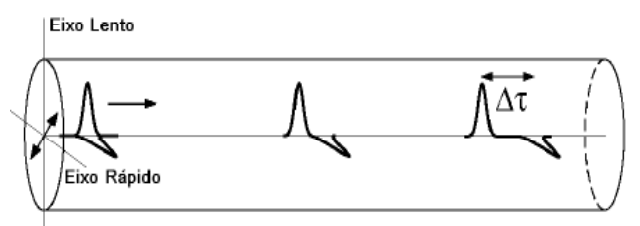

Figura 1 - Atraso de grupo diferencial

Desta maneira, a birrefringência na fibra óptica é definida como a diferença entre as constantes de propagação dos modos ortogonalmente polarizados, e pode ser escrita como:

$$
\Delta \beta=\beta_{s}-\beta_{f}=\frac{\omega}{c}\left(n_{s}-n_{f}\right)=\Delta n
$$

onde $\beta_{s}$ e $\beta_{f}$ representam as constantes de propagação do eixo lento e rápido respectivamente, $\omega$ representa a frequência angular, $c$ a velocidade da luz, $n_{s}$ e $n_{f}$ os índices de refração do eixo lento e eixo rápido respectivamente. $\mathrm{O}$ atraso de grupo diferencial pode ser calculado a partir da derivada de $1^{\text {a }}$ ordem das constantes de propagação, assim para uma pequena seção de fibra óptica, de comprimento $L$, com birrefringência constante, o $\Delta \tau$ é calculado a partir da Equação 2.

$$
\begin{gathered}
\frac{\Delta \tau}{L}=\frac{\partial}{\partial \omega}\left(\beta_{s}-\beta_{f}\right)=\frac{\Delta}{c} \\
\Delta \tau=\left(\frac{\Delta n}{c}+\frac{\omega}{c} \frac{\partial \Delta n}{\partial \omega}\right) L \\
P M D_{\text {curta_distancia }}=\frac{\Delta \tau}{L}
\end{gathered}
$$

Para um trecho longo de fibra óptica, a orientação angular dos eixos principais de birrefringência dos modos lento e rápido varia em função do local. 
Pode-se então representar o trecho longo de fibra óptica como a concatenação de trechos curtos, ou seções curtas de fibra óptica com os eixos principais de birrefringência desalinhados, conforme mostrado na Figura 2. Dessa forma, o campo elétrico de cada modo de polarização de uma seção é acoplado nos modos principais de polarização da seção seguinte. Este fenômeno é conhecido como acoplamento entre modos de polarização. Enquanto o DGD no regime de curta distância (trechos curtos) é previsível por ser considerada birrefringência constante. Não é possível considerar o mesmo fato para trechos de longa distância.

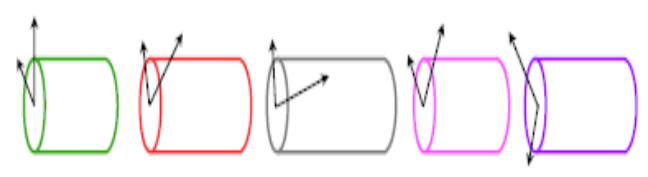

Figura 2 - Concatenação de Pequenas seções de fibra

Devido ao acoplamento modal, a birrefringência de cada seção pode ser somada ou subtraida da birrefringência total, desta forma, o DGD não acumula linearmente com o comprimento da fibra. A categorização de uma fibra em regime de curto comprimento ou grande comprimento é determinado pelo parâmetro chamado comprimento de correlação ou comprimento de acoplamento $L_{c}$ (KAMINOV,1981). O parâmetro citado é descrito como o comprimento onde a diferença de potência decai para $\left\langle P_{x}\right\rangle-\left\langle P_{y}\right\rangle=\frac{1}{e^{2}}$ (POOLE;
NAGEL, 1997).

Estatisticamente pode-se mostrar que o DGD se relaciona o comprimento de batimento e o comprimento de correlação (GALTAROSSA, 2005).

$$
\left\langle\Delta \tau^{2}\right\rangle=2\left(\Delta \tau_{b} \frac{L_{c}}{L_{b}}\right)^{2}\left(\frac{L_{c}}{L}+e^{-\frac{L}{L_{c}}}-1\right)
$$

A expressão acima pode ser simplificada para $L<L_{c}$

$$
\sqrt{\left\langle\Delta \tau^{2}\right\rangle}=\Delta \tau_{r m s}=\frac{\Delta \tau_{b} L}{L_{b}}
$$

Se for considerado $L>L_{c}$ a expressão pode ser simplificada para:
$\Delta \tau_{r m s}=\left(\frac{\Delta \tau_{b}}{L_{b}}\right) \sqrt{2 L L_{c}}$

Grandes trechos de fibras ópticas apresentam uma dependência do atraso de grupo diferencial com a raiz quadrada da distância de propagação. Desta maneira, determina-se a PMD para longa distância a partir da equação 8 .

$$
P M D_{\text {longa_distancia }}=\frac{\langle\Delta \tau\rangle}{\sqrt{L}}
$$

A PMD de $1^{\text {a }}$ ordem pode ser representada vetorialmente no espaço de Stokes (CIESLAK, 2003), por sua magnitude, $\Delta \tau$, e direção do eixo mais lento, $\vec{p}$. Desta forma o vetor PMD de primeira ordem $\Omega$ é representado por:

$$
\Omega=\Delta \tau \vec{p}
$$

A variação do vetor $\Omega$ se verifica tanto no módulo do vetor como na sua orientação na esfera de Poincaré (BALANIS, 1989), de modo que tanto o DGD quanto a orientação relativa entre a polarização do sinal e os PSPs variam no tempo e na freqüência óptica. Este fenômeno dá origem a uma distorção suplementar chamada de PMD de segunda ordem, fenômeno que vem com a variação do vetor $\Omega$ na freqüência óptica. PMD de segunda ordem é definido como a derivada da frequência do vetor dispersão $\Omega=\Delta \tau \vec{p}$. Desta forma, a PMD de segunda ordem $\Omega_{\omega}$ é dada por (SOLLER, 2005):

$$
\Omega_{\omega}=\frac{\partial \Omega}{\partial \omega}=\frac{\partial \Delta \tau}{\partial \omega} \vec{p}+\Delta \tau \frac{\partial \vec{p}}{\partial \omega}
$$

Os dois termos da equação acima geralmente são tratados separadamente, desmembrando a PMD de segunda ordem em uma componente perpendicular e outra paralela.

$$
\begin{aligned}
& \Omega_{\omega I I}=\frac{\partial \Delta \tau}{\partial \omega} \vec{p} \\
& \Omega_{\omega \perp}=\Delta \tau \frac{\partial \vec{p}}{\partial \omega}
\end{aligned}
$$




\section{MÉTODOS DE MEDIDA DA PMD}

Atualmente diversas técnicas são capazes de calcular a PMD. A maioria dessas técnicas determina o atraso de grupo diferencial entre os estados de polarização na saída da fibra, recorrendo a métodos que podem ser tanto no domínio temporal como no domínio da frequência. Neste trabalho serão abordados apenas dois métodos, os quais foram utilizados para fazer as medidas. São eles: Método interferométrico e método polarimétrico.

\subsection{MÉTODO INTERFEROMÉTRICO}

O método interferométrico funciona a base do fenômeno de interferência da luz. Um interferômetro, no caso específico, o de Michelson, é capaz de determinar o DGD induzindo um pequeno atraso em parte do feixe que chega ao receptor óptico. Através desta divisão de feixe e indução de atraso, é possível determinar sua função de autocorrelação dos dois sinais derivados da mesma fonte óptica. Para que ocorra o fenômeno de interferência, é necessário que o atraso gerado pelo espelho móvel entre os dois sinais seja menor que o tempo de coerência da fonte, que é definido por $\frac{\lambda^{2}}{c \Delta \lambda^{2}}$ onde $\Delta \lambda$ é a largura espectral da fonte óptica utilizada no experimento e $\lambda$ é o comprimento de onda central desta fonte. Através de um interferograma, que neste caso é a função de autocorrelação do campo elétrico, é possível calcular o DGD. Na figura abaixo verifica-se um esquema básico de medida de DGD utilizando um interferômetro de Michelson, tanto para fibras HI-BI (fibras de alta birrefringência) como para fibras standard (WILLIAMS, 2002), (GALTAROSSA, 2005).
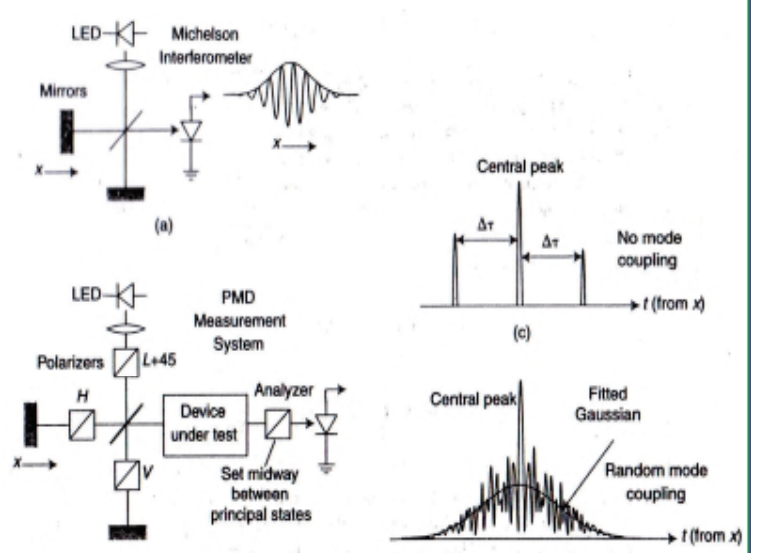

Figura 3 - Modelo do método interferométrico
$\mathrm{Na}$ Figura 4, é possível visualizar o interferograma (função de autocorrelação) do campo elétrico da fonte. $\mathrm{O}$ pico posicionado em 0 é a função de coerência da fonte e não contém informaçôes sobre a birrenfringência. $\mathrm{O}$ atraso de grupo diferencial pode ser simplesmente encarado como a distância entre o lobulo central e o lobulo lateral

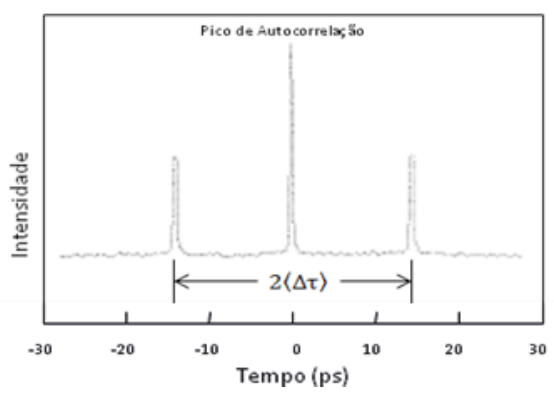

Figura 4 - Pico de autocorrelação e picos laterais

\subsection{MÉTODO POLARIMÉTRICO}

Para a caracterização completa da PMD, válida sobre qualquer condição de acoplamento, define-se um vetor $\Omega$ cujo módulo é o valor do DGD e sua direção caracteriza os PSP na esfera de Poincaré. Este vetor varia no tempo devido à micro-variações das condições externas da fibra e com o comprimento de onda do sinal óptico, característica esta que está diretamente ligada ao fenômeno da birrefringência.

$O$ vetor de dispersão de polarização $\Omega$ é relacionado à mudança ao estado de polarização de saída S como (WILLIAMS, 2002), (GALTAROSSA, 2005).

$$
\frac{\partial s}{\partial \omega}=\Omega \times S
$$

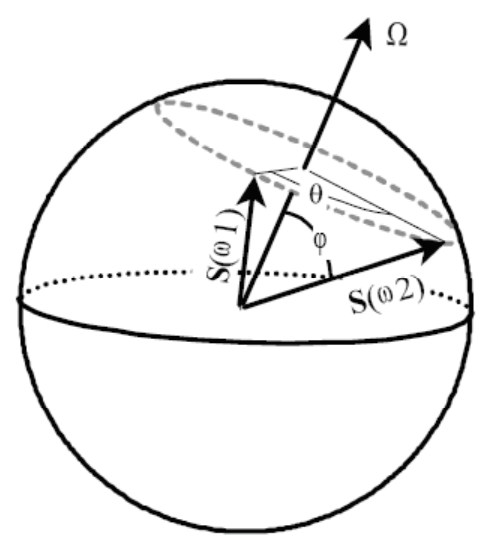

Figura 5 - Representação do vetor dispersão de polarização $\Omega$ e a saída $S$ na esfera de Poicaré. 
A medida dos PSP é feita lançando a luz em três diferentes polarizações, geralmente $0^{\circ}, 45^{\circ} \mathrm{e}$ $90^{\circ}$. Para determinar o atraso de grupo por este método faz-se o produto das matrizes das três polarizações em duas frequências ópticas distintas. $\mathrm{Na}$ esfera de poincaré é possível verificar que o estado de polarização de saída $S$ varia quando a freqüência é alterada de $\omega_{1}$ para $\omega_{2}$.

O DGD pode ser calculado a partir da equação 13 , onde $\theta$ é definido como o ângulo de rotação entre os estados de polarização de saída.

$\left|\frac{\partial \theta}{\partial \omega}\right|=|\Omega|=\Delta \tau(14)$

Dentre as técnicas polarimétricas existentes, três delas são muito citadas em diversas literaturas.

- Jones Matrix Eigen analysis (CLARK, 1946), (BRIAN, 1992)

- Muller Matrix Method (BRIAN, 1992).

- Poincaré Sphere analysis (GORDON; KOGELNIK, 2000).

\section{MONTAGEM EXPERIMENTAL PARA AS MEDIDAS DA PMD}

As fibras ópticas possuem uma grande sensibilidade térmica sendo que pequenas variações de temperatura causam grandes variações no estado de polarização, consequentemente no DGD. Desta forma, foram realizadas medidas da PMD na temperatura ambiente e logo em seguida aumentou-se a temperatura para constatar a influência da temperatura em relação a PMD. Dois tipos de equipamentos foram usados: Método interferométrico no domínio temporal (EXFO FTB5500) e método polarimétrico no domínio da freqüência (Adaptif Photonics A2000). Na figura 6 é visto o setup experimental.

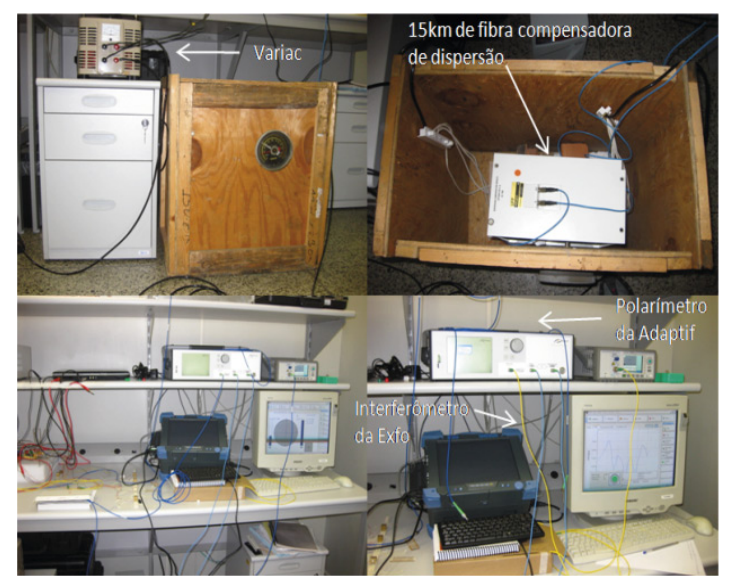

Figura 6 - Fotos do setup experimental

\subsection{EQUIPAMENTO EXFO FTB-5500}

Este equipamento utiliza o fenômeno da interferência da luz para determinar a PMD média no domínio temporal entre os comprimentos de onda: 1527.68 - $1627.43 \mathrm{~nm}$. Abaixo segue o setup experimental para medida da PMD, na temperatura ambiente, utilizando uma fonte de luz polarizada conectorizada com conectores APC a um carretel de $15 \mathrm{~km}$ de fibra compensadora de dispersão e ao interferômentro da Exfo.

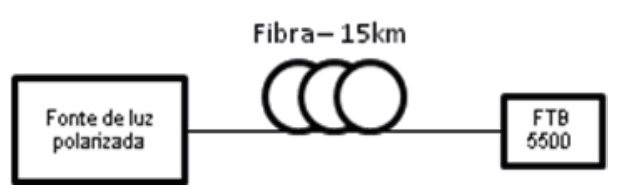

Figura 7 - Diagrama do setup experimental

No setup experimental da figura 8 foi acrescentado um gerador de tensão (Variac) em série com uma resistência. Estes materiais foram colocados dentro de uma caixa de madeira com um termômetro industrial que estava localizado no centro do carretel da fibra. $\mathrm{O}$ variac teve a função de gerar corrente $\mathrm{AC}$ para que a resistência transformasse em calor, simulando uma variação climática. Com o termômetro posicionado próximo ao centro do carretel da fibra, pode-se supor que a temperatura da fibra seria muito próxima à temperatura no centro do carretel.

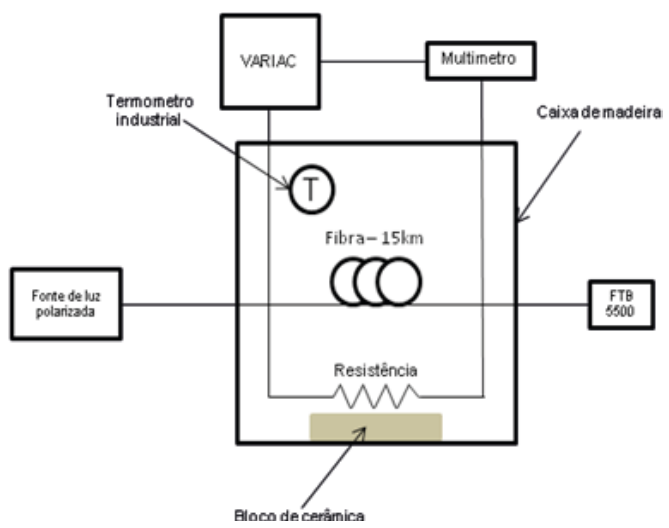

Figura 8 - Setup experimental utilizando variac em série com uma resistência dentro da caixa de madeira

\subsubsection{Meddidas na temperatura ambiente}

Foram realizadas 20 medidas na temperatura ambiente $\left(23^{\circ} \mathrm{C}\right)$ no enlace de fibra compensadora de dispersão de $15 \mathrm{~km}$. Na Figura 9 
pode-se visualizar a tela do equipamento (EXFO FTB-5500) em uma das medidas realizadas nessa temperatura. A PMD média foi de 0,234 ps. Vale destacar que as demais medidas tiveram valores bem aproximados pois não houve variações significativas no ambiente de teste.

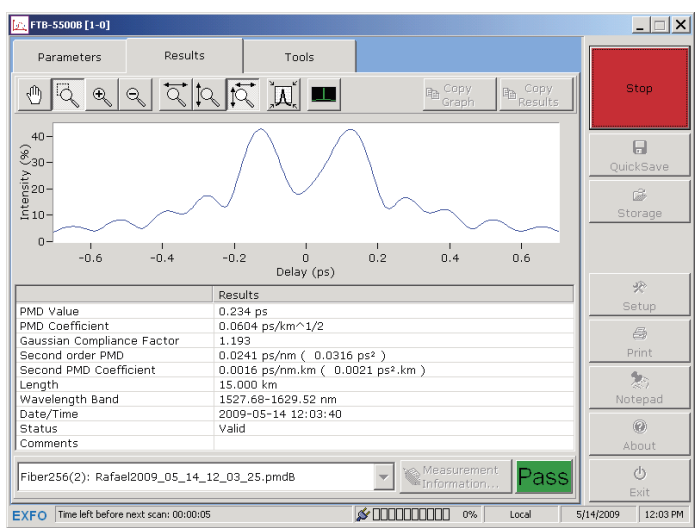

Figura 9 - Tela do EXFO FTB-5500 para uma das 20 medidas de PMD realizadas em um carretel de fibra DCF de $15 \mathrm{Km}$

\subsubsection{Temperatura em torno de $45^{\circ}$ celsius}

O multímetro mostrado na Figura 8, teve a função de controlar a corrente que estava sendo aplicada na resistência, que não deveria passar de 15 A para não exceder o limite de dissipação térmica. Após a estabilização térmica em $45^{\circ} \mathrm{C}$, verificada pela manutenção da temperatura no interior $\mathrm{da}$ caixa por um longo período de tempo (Aproximadamente 1 hora), iniciaram-se as medidas. $\mathrm{Na} \mathrm{Fi-}$ gura 10 é mostrado o gráfico com os dados obtidos para as 20 medidas consecutivas, com intervalo de 30 segundos. Vale destacar que a PMD variou mais de $100 \%$ em relação as medidas da temperatura ambiente e chegou a valores superiores a 0,6 ps.

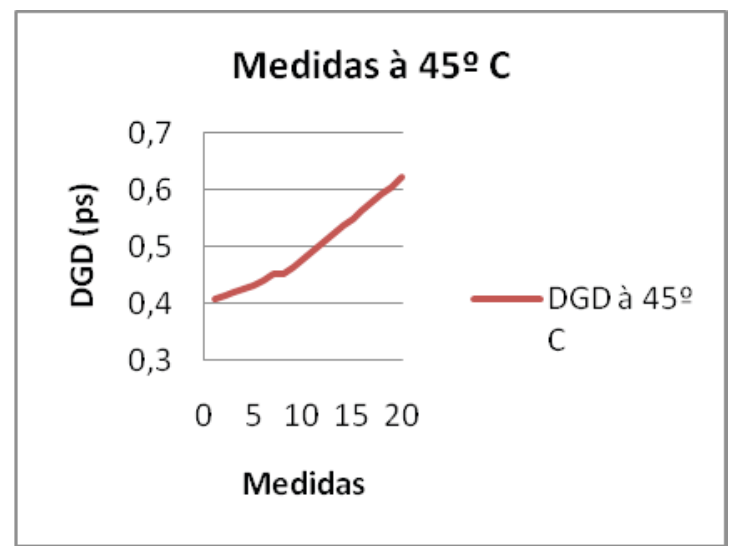

Figura 10 - Gráfico DGD x Medidas para o método interferométrico a $45^{\circ} \mathrm{C}$

\subsection{EQUIPAMENTO ADAPTIF PHOTONICS A2000}

Este equipamento utiliza a técnica polarimétrica para medir a PMD no domínio da freqüência e faz a varredura entre os comprimentos de onda de 1522,2 até $1630,3 \mathrm{~nm}$. Uma importante diferença entre o método interferométrico e o polarimétrico, é que o método polarimétrico além de conseguir medir a PMD média em uma faixa de frequência, também consegue determinar a PMD em um comprimento de onda específico. Esta medida é extremamente importante para sistemas WDM, pois é possível caracterizar individualmente cada canal óptico. A Figura 11 mostra o setup experimental utilizando o equipamento A2000 da Adaptif Photonics.

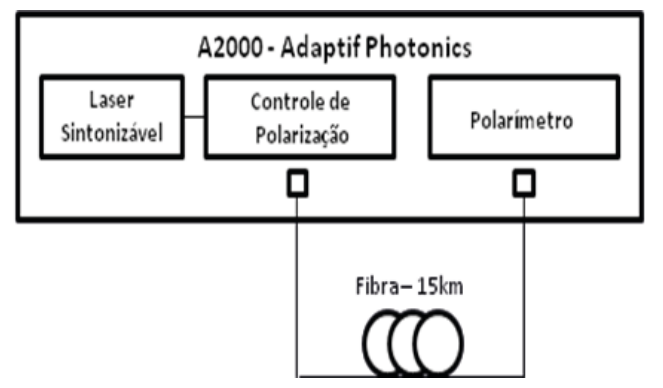

Figura 11 - Setup experimental para amedida de PMD utilizando o equipamento da Adaptif Photonics

$\mathrm{Na}$ figura 12 é possivel visualizar o estado de polarização na esfera de poincaré, os parâmetros de Stolkes S1, S2 e S3 e o degree of polarization no equipamento da Adaptif Photonics. Estes parâmetros variam constantemente ao longo do trajeto óptico desde que sofram alteração na fibra óptica, um simples toque na fibra altera bastante o ponto que é marcado na esfera de poincaré. Se a fibra permanecer na mesma condição inicial, tende a variar pouco.

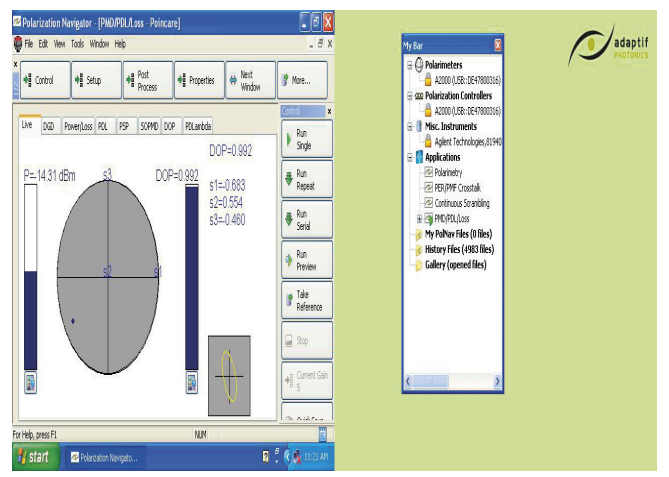

Figura 12 - Visualização da esfera de Poincaré no software Polarization Navigator da Adaptif com os respectivos valores dos parâmetros de Stokes, DOP e potência em $\mathrm{dBm}$ 


\subsubsection{Medidas na temperatura ambiente}

Foram realizadas medidas do DGD com o equipamento A2000 da Adaptif Photonics. Inicialmente, assim como feito na seção 4.1.1 usando o equipamento da EXFO, as medidas da DGD foram realizadas na temperatura ambiente. A Figura 13 mostra a medida do DGD, em função do comprimento de onda. Pode-se verificar que o DGD varia significativamente com o comprimento de onda. Porém, o DGD médio está de acordo com o encontrado no método interferométrico, e usando o equipamento da EXFO.

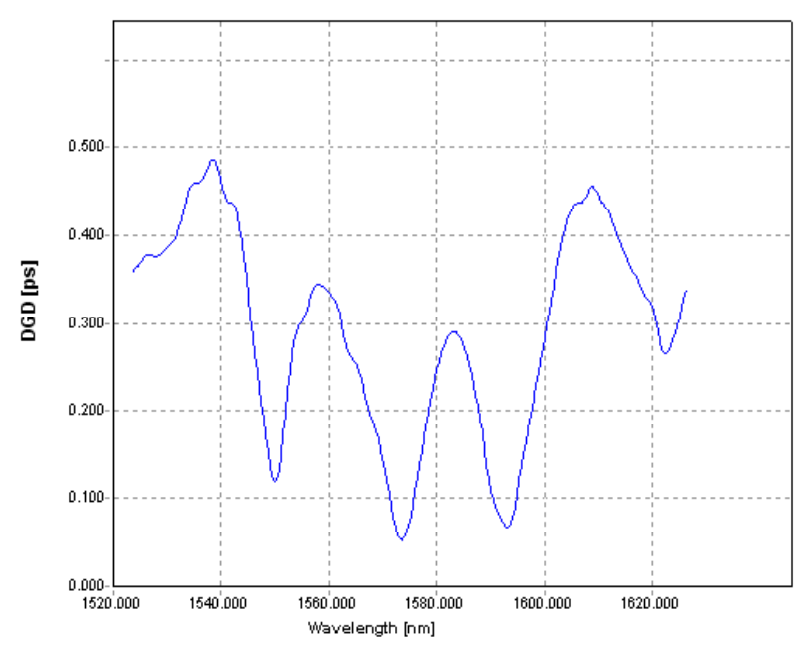

Figura 13 - Medida da DGD versus comprimento de onda com PMD média de 0.291 [ps]

O setup experimental foi o mesmo mostrado na Figura 7, porém utilizando o equipamento da Adaptif Photonics.

\subsubsection{Temperatura em torno de $45^{\circ}$ celsius}

Logo após terem sido realizadas as medidas de DGD na temperatura de $45^{\circ} \mathrm{C}$ com o método interferométrico, trocou-se o instrumento de teste pelo A2000 da Adaptif Photonics. A Figura 14 mostra a variação do DGD, em função do comprimento de onda, para a temperatura de $45^{\circ} \mathrm{C}$. Não foi possível ter um equilíbrio térmico adequado, porém constatou-se a influência da temperatura no DGD versus comprimento de onda.

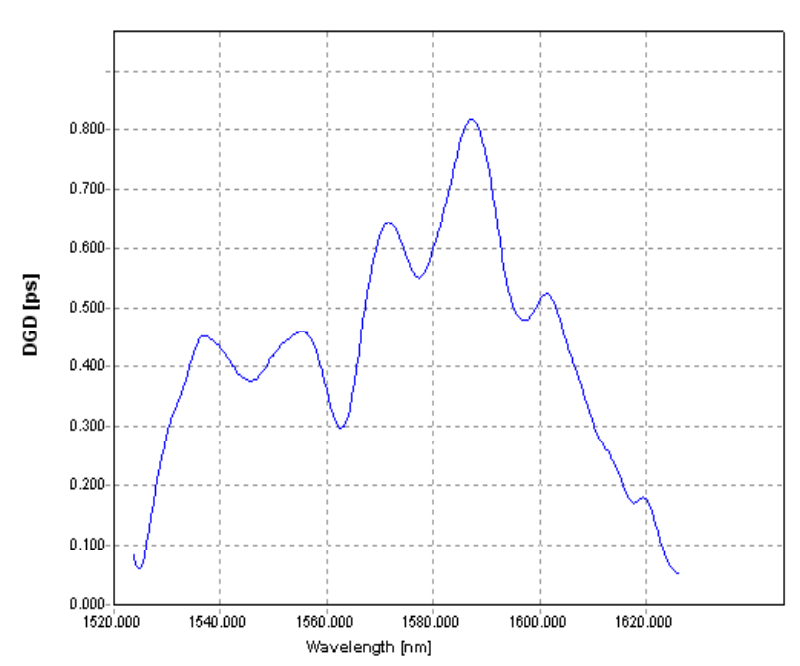

Figura 14 - Medida da DGD versus comprimento de onda na temperatura proximada de $45^{\circ} \mathrm{C}$ com uma PMD média de 0.424 [ps]

\section{CONCLUSÔES}

Como foi visto nas primeiras seçôes deste trabalho, a PMD é um fenômeno aleatório, e para sua compensação é necessário conhecer bem as características de sua variação em função dos diversos parâmetros encontrados em sistemas de comunicação. Para o controle da PMD, deve-se iniciar por definir um método de medida que forneça informações em todo o espectro de interesse dos sistemas DWDM, e em todas as bandas ópticas. O método interferométrico é capaz de medir apenas a PMD média, dentro de uma faixa de comprimentos de onda. Portanto, não é muito útil para sistemas DWDM, pois cada canal possui uma característica específica. Já o método polarimétrico, é capaz de medir a PMD em função do comprimento de onda, sendo mais adequado para a tecnologia DWDM. Foi constatado que a temperatura tem uma grande influência no DGD, e que o mesmo varia significativamente quando a temperatura é variada de $23^{\circ} \mathrm{C}$ para $45^{\circ} \mathrm{C}$, que foram os limites experimentais usados no experimento. Desta forma foi verificado que um importante parâmetro para a caracterização da PMD é a temperatura, que no presente trabalho variou mais de $100 \%$ dentro dos limites experimentais estabelecidos. 


\section{BIBLIOGRAFIA}

Kaminov, I. P., "Polarization in optical fibers," IEEE J. Quant. Electron., QE-17, 15-22 (1981).

Poole, C. D. and J. A. Nagel, "Polarization effects in lightwave systems," In Optical Fiber Telecommunication IIIA, I.P, Kaminov and T.L. Koch, eds.(Academic Press, CA, 1997). Pp. 114-161.

Soller, Brian J. "Second Order PMD in Optical Components", Luna Technologies, 2020 Kraft Drive, Suite 2000, Blacksburg, VA 24060 May 13, 2005.

P.A. Williams," PMD Measurement Techniques - Avoiding Measurement Pitfalls," Venice Italy, 24-26 June, 2002

Galtarossa, Andrea. Polarization Mode Dispersion. Department Information. USA, 2005.

R. Clark Jones, "A New Calculus for the Treatment of Optical Systems: VI. Experimental Determination of the Matrix," J.O.S.A. 37, 110-112 (1946).
Brian L. Heffner, "Recent Progress in Polarization Measurement Techniques," HPL-92-72, June, 1992.

J. P. Gordon and H. Kogelnik. PMD fundamentals: Polarization mode dispersion in optical fibers. February 2, 2000.

P. S. André e J. L. Pinto. “Birrefringência e Dispersão Devido aos Modos de Polarização em Fibras Ópticas”. Revista do Detua, Vol. 3, no 5, Junho 2002.

J. Arlindo. " Teoria da Dispersão dos Modos de Polarização - PMD," Tese de Mestrado da PUCRio, Julho de 2003.

G. J. Foschini and C. D. Poole, "Statistical theory of polarization dispersion in single mode fibers," $\mathrm{J}$. Lightwave Technol., vol. 9, pp. 1439-1456, 1991.

Ferreira, Janaina. Simulações do comportamento temporal da dispersão dos modos de polarização. Tese de Doutorado, abril 2007.

Mello, Fernanda. Penalidades devido a multiplexação óptica no domìnio do tempo em 40Gbps. Tese de Doutorado da PUC-Rio, Fevereiro de 2002. 